Commentary

\title{
The Need for Nutrient Efficient Crop Varieties
}

Vinod K K

Indian Agricultural Research Institute, Division of Genetics, New Delhi 110012

Corresponding address:

Rice Breeding and Genetics Research Centre, Indian Agricultural Research Institute, Aduthurai 612101, Tamil Nadu, INDIA.

Tel: +914352470308

E-mail: kkvinod@iari.res.in 


\section{The Need for Nutrient Efficient Crop Varieties}

\section{Vinod K K}

Indian Agricultural Research Institute, Division of Genetics, Rice Breeding and Genetics

Research Centre, Aduthurai 612101, INDIA

Since the birth of genetics as a science almost one and a half century ago with Gregor Mendel's classical pea hybridization experiments, plant breeding has taken leaps and bounds to reach modern day crop varieties that sustain humanity. Genetic improvement of crop varieties has scaled from introduction of 'good' and 'tasty' food species form wild and the selection for the 'best' crops under domestication to vistas of hybridization for developing superior recombinants and robust hybrids. Development of genetics, always paved new ways and means for crop improvement. Past the discovery of DNA and more clearer understanding of the genes, genetic manipulation has turned to molecular levels from mere visual (qualitative) and measurable (quantitative) selection. The growing food needs for the expanding world population, has always been driving the need for sustained development of high yielding crop varieties.

\section{Diminishing nutrient sources on earth}

Corresponding to the development of better crop varieties, agricultural practices had taken new dimensions and methods to support large scale food production. The rapid developments in crop management and crop improvement which ran mutually complimenting paved way to 'green revolution' in several key food crops which almost eradicated famines from the face of earth. However, the best agro-management always required large quantum of agronomic inputs such as fertilizers. The modern varieties need high positive nutrient balance in the soil to throw their best yields. Currently, indiscriminate chemical inputs into agriculture either as fertilizers or pesticides has been recognized as an environmental hazard, with respect to varying adverse consequences such as residual toxicity, pest resurgence, development of pathotypes through inadvertent mutations, biohazards, eutrophication, volatilization, acid rains, ozone depletion and so forth. Another major concern with the major fertilizers is that almost all of them are resourced from natural rocks and deposits that are non-renewable. Continuous mining of these resources is depleting their natural reserves so fast that none of these may exist beyond next 500-600 years.

\section{Fertilizer overdependence may turn costly}

Crop plants require sixteen elements for their survival and growth, of which three essential elements, carbon, hydrogen and oxygen are sourced through water and air. Of the remaining thirteen, three mineral elements, nitrogen, phosphorus and potassium are required in large quantities (major nutrients), and others are either required in minor 
quantities (secondary nutrients) or in micro quantities (micronutrients). Nitrogen fertilizers are required by crop plants in large quantities followed by phosphatic and potash fertilizers. Chemical synthesis of nitrogen fertilizers uses ammonia produced through Haber-Bosch process that consumes huge quantity of petroleum and natural gas, the major natural non-renewable energy reserve. Whereas, phosphatic fertilizers are exclusively sourced from phosphate rock reserves and potassium fertilizers depend on large deposits of potassium salts. Overdependence of mineral fertilizers for agricultural production envisions problems such as, (a) soil nutrient deficiency caused by continuous crop removal and fixation through soil chemical processes, (b) loss of significant amount of nutrients through crop harvests and food chain that are non-recycled, (c) significant loss of applied nutrients through leaching and volatilization due to poor nutrient use efficiency of crops, (d) nutrient pollution in the water bodies resulting in eutrophication and life-threatening algal bloom toxicity in the aquatic ecosystems, (e) atmospheric pollution causing ozone depletion and green-house effects, (f) faster depletion of nonrenewable natural resources for fertilizer production, (g) geographic confinement of most of the natural fertilizer resources (especially phosphate rock) instill global overdependence on few countries that may create unhealthy monopolistic situation, (h) booming fertilizer costs in the wake of diminishing production and increasing demand is making cost of cultivation to go up, (i) unavailability and constrained affordability of fertilizers by the marginal farmers are rendering more agricultural areas nutrient deficient worldwide, and finally $(j)$ unavailability of alternate fertilizer resources other than the natural sources is a serious threat to the future sustenance of agriculture.

\section{Sustaining the crop production may require low fertilizer inputs}

To sustain agriculture in future, we need to preserve environmental and soil health by preventing undesirable nutrient drainage from soil and also need to prolong the supply of natural nutrient resources. This implies that there is an immediate need for reduction in soil nutrient input. Although skipping chemical fertilization altogether may not be possible in modern agriculture, input reduction is a feasible alternative. Modern organic agricultural systems advocate skipping of inorganic fertilization, with the objectives of maintaining biodiversity at soil, crop, field, seasonal and landscape level with a greater focus on integration of crop and livestock production systems and by using organic fertlization ${ }^{1}$. However, in the current agricultural scenario, fertilizer reduction may lead to serious crop production loss and may accelerate negative nutrient balance in soil. Therefore, a more sustainable approach is to develop crop varieties that respond well to low fertilizer doses and are high uptake efficient even under reduced nutrient conditions. Use of low input happy varieties can go in tandem with fertilizer reduction and organic fertilization. The reduced availability of bio-available nutrients in the soil, a common phenomenon under soil nutrient deficiency, occurs either through fixation of available nutrients into bio-unavailable forms or due to depletion. Either cases levy tremendous stress of nutrient starvation in plants. Ability of plants to withstand nutrient starvation is leveraged by a variety of mechanisms such as deeper and wider root system for nutrient harvest from soil, root exudations to solubilize fixed nutrients into available forms, root 
microfloral symbiosis to promote nutrient availability and better nutrient transport systems ${ }^{2}$.

\section{Seeking the genetic route}

Crop responses to nutrients and their genetics differ fundamentally under nutrient sufficiency and deficiency. Nutrient sufficiency does not produce any stress on the plants unless it is excess to the toxic levels, whereas nutrient starvation does incite a stress response. Estimates of genotype response to applied nutrients, nutrient use efficiency (NtUE) is basically done under nutrient sufficient conditions therefore does not have any stress response component attached to it. Conventionally, NtUE is defined as the quantum of economic output realized per unit nutrient applied. Thus, scope of this estimate is confined to nutrient responding genotypes of the high yielding category because it cannot rank genotypes that are low nutrient tolerant. Therefore, NtUE is a term that should be limited to nutrient sufficient conditions, and for nutrient starved situations terms such as 'low nutrient tolerance', 'nutrient starvation tolerance' or 'nutrient efficiency (NtE)' may be more appropriate. The term NtE is suggested because the low nutrient response and its consequences fundamentally include all mechanisms of nutrient homeostasis comprising of low nutrient signaling and responses, active recovery, uptake and assimilation. May be new indices are needed to define various components of NtE and to identify the genetic mechanisms underlying low nutrient tolerance. This should also take into consideration of the soil nutrient status before and after the application of fertilizer, temporal soil nutrient fluctuation and plant biomass assimilation rate. In systems such as organic farming wherein organic fertilization alone is done, temporal soil nutrient fluctuations may be very important in defining the $\mathrm{NtE}$, because it will help in accommodating the effects of slow release organic fertilizers. Further, nutrient fluctuations also occur when fixed soil nutrients are released either through root solubilization activity or by native root microbial symbiosis or through addition of biofertilizers. Other components of NtE are maintenance of photosynthesis, nutrient-uptake, nutrient-utilization and translocation under nutrient stress. Hence quantum of economic output yielded per unit net soil available nutrient throughout the cropping season may be a better index in identifying low nutrient tolerant genotypes.

Plant nutrient homeostasis depends on uptake and utilization processes, which are different for different nutrients and vary between phonological stages. At this point, both NtUE and NtE share common components such as uptake efficiency (UpE) and utilization efficiency (UtE). UpE is predominantly related to rhizosphere processes regulating better foraging for nutrients in the soil, in association with efficient uptake and transport mechanisms of nutrient ions into the plants. It is apparently important that UpE under relatively lower nutrient availability is to be considered as an important trait for developing nutrient efficient varieties. Balanced internal nutrient mobilization and assimilation is essential to maintain UtE, and nutrient efficient genotypes should therefore have efficient uptake in harmony with internal assimilation processes. Considerable genetic variations are reported for low nutrient responses in various crops, 
although a clear practical differentiation between UpE and UtE is still lacking. Therefore it is essential to develop phenotyping protocols, agronomic, biochemical and physiological to distinguish UpE and UtE. Since the genetics of NtUE and NtE are different, it may be possible to combine both the traits to develop widely adapted varieties that may perform well under both nutrient deficient and sufficient conditions, although not at the same performance level.

Selection for NtUE in crop plants has been hugely successful and is responsible for green revolution in major staple crops. However in this process, many low nutrient responsive genes got inadvertently unselected and lost. Therefore, sourcing of nutrient starvation tolerance genes from the modern cultivated high yielding germplasm is likely to go unsuccessful and therefore old traditional varieties and landraces may be potential sources of such genes. Several instances of better performance of landraces and traditional varieties under low nutrient soils have been reported in rice, wheat, tomato, cauliflower etc., than the modern cultivars. Therefore, it is essential that older germplasm be screened for nutrient starvation tolerance genes.

\section{Future is not bleak}

Unlike that of breeding for NtUE, recent studies show that NtE has very low heritability and is likely to give little progress through conventional breeding. Hence more direct genetic approaches such as marker assisted selection or genetic transformation may be required to manipulate $\mathrm{NtE}$ in crops. Although indirect selection is advocated in conventional breeding under low heritability situations, to take advantage of correlated selection responses under contrasting environments ${ }^{3}$, relative gain towards $\mathrm{NtE}$ may likely to be low as most of the low nutrient response genes are already lost in the elite germplasm. Molecular dissection of phenotype performance is now possible in almost all major food crops that may aid largely in identifying genomic regions that are responding to low nutrient exposure. Several such regions and QTLs have been reported in crops like rice, wheat, maize, barley etc. Molecular isolation of large effect QTLs for phosphorus starvation tolerance and the gene responsible for the root proliferation under low phosphorus conditions, PSTOL1 (phosphorus starvation tolerance 1) has been reported in rice $^{4}$. Identified from a traditional indica variety Kasalath ${ }^{5}$, the quantitative trait locus (QTL) containing PSTOL1, known as Pup1, has already been used for marker assisted transfer of the gene imparting low phosphorus tolerance in sensitive varieties ${ }^{6}$. Likewise, many QTLs for low nitrogen response have also been mapped in major staple crops, rice and wheat. Apart from nitrogen and phosphorus, works are progressing for identifying tolerance for reduced levels of other nutrient elements such as zinc, sulphur and potassium. It is likely that several improved varieties with better NtE would be developed using modern molecular breeding technology in the days to come.

\section{References}

1. van Bueren, E.T.L., Jones, S.S., Tamm, L., Murphy, K.M., Myers, J.R., Leifert, C. and Messmer, M. M., The need to breed crop varieties suitable for organic farming, using 
wheat, tomato and broccoli as examples: A review. NJAS - Wagen. J. Life Sc., 2011, 58, s 193-205

2. Gregory, P. J., Crop root systems and nutrient uptake from soils. In The molecular and physiological basis of nutrient use efficiency in crops (ed. Hawkesford M. J. and Barraclough, P.), John Wiley \& Sons, 2011, pp. 21-45.

3. Falconer, D.S. and Mackay, T. F. C., Introduction to Quantitative Genetics, 4/e. Longman, 1996.

4. Gamuyao, R., Chin, J. H., Pariasca-Tanaka, J., Pesaresi, P., Dalid, C., Slamet-Loedin, I., Tecson-Mendoza, E. M., Wissuwa, M. and Heuer, S., The protein kinase Pstol1 from traditional rice confers tolerance of phosphorus deficiency. Nature, 2012, 488, 535539

5. Wissuwa, M., Yano, M. and Ae, N., Mapping of QTLs for phosphorus-deficiency tolerance in rice (Oryza sativa L.). Theor. Appl. Genet., 1998, 97, 777-783

6. Chin, J. H., Gamuyao, R., Dalid, C., Bustamam, M., Prasetiyono, J., Moeljopawiro, S., Wissuwa, M. and Heuer, S., Developing rice with high yield under phosphorus deficiency: Pup1 sequence to application. Plant Physiol., 2011, 156, 1202-1216. 